\title{
Propuesta de sistema de gestión inteligente basado en IoT para hidroponia
}

\author{
Evelyn Gutierrez Leon ${ }^{1}$, Jorge Erik Montiel Arguijo ${ }^{1}$, Chadwick Carreto Arellano ${ }^{2}$, \\ Felipe Rolando Menchaca García ${ }^{1}$ \\ ${ }^{1}$ Instituto Politécnico Nacional, Escuela Superior de Ingeniería Mecánica y Eléctrica Unidad \\ Zacatenco, Ciudad de México, México \\ ${ }^{2}$ Instituto Politécnico Nacional, Escuela Superior de Cómputo, Ciudad de México, México \\ evelyngleon@hotmail.com, jerikmontiel.isc@gmail.com, \\ ccarretoaeipn.mx, fmenchacegmail.com
}

\begin{abstract}
Resumen. Este artículo propone el diseño de un sistema de gestión inteligente basado en el paradigma de internet de las cosas (IoT) para el caso de uso de cultivos hidropónico. Se propone la arquitectura de red y diseño de prototipos enfocados a obtener la información en "tiempo real" de los parámetros clave que desarrollo de los cultivos hidropónicos de escala semi-comercial y de autoconsumo, así como la primera versión de la interfaz gráfica para dispositivos móviles que permitirán la gestión del cultivo y toma de decisiones. La premisa del proyecto es el desarrollo de una solución modular, escalable, de bajo costo, fácil instalación y uso.
\end{abstract}

Palabras clave: internet de las cosas, agricultura inteligente, IoT, redes inalámbricas.

\section{Proposed Smart Management System Based on IoT for Hydroponics}

\begin{abstract}
This article proposes the design of an intelligent management system based on the Internet of Things (IoT) paradigm for the case of hydroponic crop use. The network architecture and design of prototypes are proposed, focused on obtaining the information in "real time" of the key parameters that develop the hydroponic crops of semi-commercial and self-consumption scale, as well as the first version of the graphic interface for mobile devices that will allow crop management and decision making. The premise of the project is the development of a modular, scalable, low-cost solution, easy installation and use.
\end{abstract}

Keywords: internet of things, smart agriculture, IoT, wireless networks.

\section{Introducción}

La agricultura es la tercera actividad económica en el país, cerca de 22 millones de hectáreas son dedicadas al cultivo, de las cuales, el 75.67\% implementan algún nivel de mecanización y de éstos $64.92 \%$ son cultivos de temporal [1]. 
De la población agrícola, aproximadamente el $35 \%$ de los agricultores se encuentra en el sector micro y pequeña empresa con lazos en el mercado interno y el $50 \%$ produce para autoconsumo. A pesar de existir diversas soluciones importadas para el campo agroalimentario tales como los invernaderos, equipos de monitoreo, de control climático y de riego, que ofrecen múltiples ventajas en la producción, éstos son enfocados a productores intensivos de escala industrial, los cuales requieren una alta inversión inicial y personal altamente calificado en la tecnología para su instalación, operación y mantenimiento, por lo cual, su implementación no ha sido generalizada para productores de los sectores pequeña, micro empresa y huertos de autoconsumo, quienes son más vulnerables ante la variabilidad y el cambio climático[2].

Dicho lo anterior, el principal reto en el campo agrícola es satisfacer la demanda de producción de alimentos manteniendo los niveles sustentables de los recursos naturales [3], por lo cual, la implementación de la ciencia y nuevas tecnologías en la agricultura es pieza clave para diseñar soluciones de bajo impacto ambiental. En donde, el desarrollo de soluciones basadas en Internet de las cosas (IoT) y la agricultura de precisión (AP), han creado un amplio panorama de desarrollo de dispositivos y equipos que satisfagan las necesidades de monitoreo y control de las diversas etapas dentro de la campaña de cultivo, las cuales han sido implementadas y adoptadas con gran ímpetu en múltiples países desarrollados [4].

La AP también es conocida como agricultura inteligente (o Smart Farming) dado que se caracteriza por el monitoreo constante a través del uso de diversas tecnologías de la Información y Comunicaciones (TIC) así como de la automatización, aunando el conocimiento científico y tecnológico con las metodologías agrícolas. En donde el papel de Internet de las cosas (IoT) ha sido ampliamente destacado [5 y 6].

El presente artículo se enfoca a describir el diseño del sistema enfocado en la primera etapa de diseño de dispositivos IoT para captación de la mayor cantidad de información posible para que en una futura fase, implementar algoritmos avanzados para procesar la información y realizar la toma de decisiones y autoconfiguración de los parámetros clave que influyen en el desarrollo del cultivo [7 y 8].

\section{Diseño del sistema}

Las características y requerimientos de los sistemas agrícolas semicomerciales y de autoconsumo suelen ser muy variables: la extensión del cultivo, la distribución de las plantas, tipo de sistema hidropónico. Por lo cual, para satisfacer las necesidades de monitoreo, control y gestión de los sistemas hidropónicos antes mencionados, es necesario que la solución cumpla las siguientes características: modular, flexible, configurable y de bajo costo.

Se propone un sistema en el cual, se conjugan conocimientos y desarrollos de la agricultura de precisión e Internet de las cosas, con la finalidad de reducir costos en el desarrollo de la solución, pero ofreciendo las capacidades de gestión de cultivos similares a las soluciones industriales y comerciales actualmente existentes (Fig. 1). 


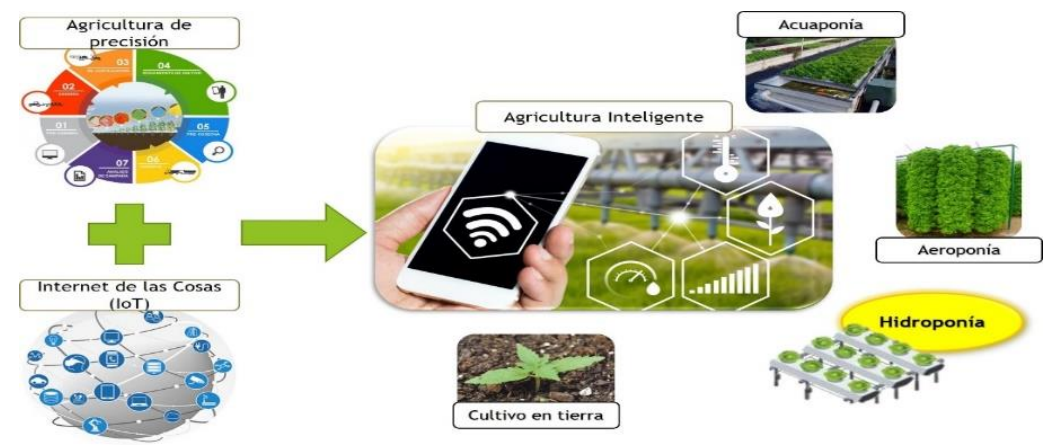

Fig. 1. Esquema de la solución de Agricultura inteligente basada en IoT y agricultura de precisión.

\subsection{Arquitectura de red}

Inicialmente la arquitectura de red fue basada en el modelo de referencia definido por el grupo de trabajo P2413 del IEEE-SA [9], al cual le fue agregado una cuarta capa (intermedia a la capa de red y la capa de aplicación) llamada capa de procesamiento de datos. (Fig. 2)

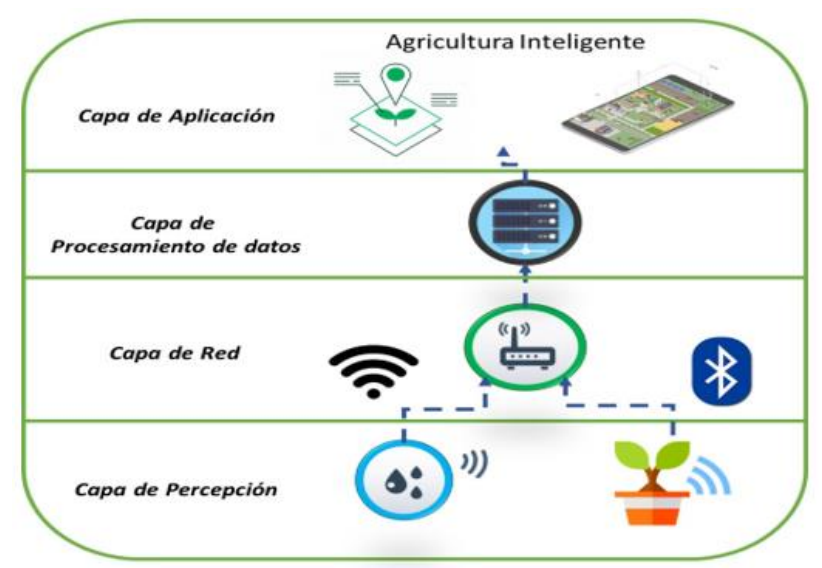

Fig. 2. Arquitectura de referencia IoT de cuatro capas para el sistema de gestión inteligente para cultivos hidropónicos "Pouani".

\subsection{Capa de percepción}

En la capa de percepción se encuentran los dispositivos capacitados por una serie de sensores y actuadores, dedicados a la tarea de adquirir información del entorno del cultivo dentro del invernadero y modificarla. Dentro de esta capa se definen dos tipos de dispositivos: Nodo Sensor y Nodo de Riego. El diseño de los nodos se caracteriza por constar de los bloques o unidades de alimentación (por ejemplo, baterías), percepción (sensores y actuadores), procesamiento $(\mu C)$ y de comunicación (transceptores) cada uno (Fig. 3-5). Estos nodos a su vez son gestionados dentro del invernadero por el nodo Maestro. 


\section{Nodo Sensor}

La tarea principal del Nodo Sensor (Fig. 3) es recolectar la información del entorno a través de diversos parámetros clave que influyen en el desarrollo del cultivo, tales como la Iluminación (lx), temperatura $\left({ }^{\circ} \mathrm{C}\right)$, Humedad $(\% \mathrm{HR})$ y temperatura de la Solución Nutritiva (SN). Con la finalidad de obtener datos con mayor precisión, se pretende ubicar el nodo Sensor en el mismo módulo hidropónico en donde se coloca las plantas.

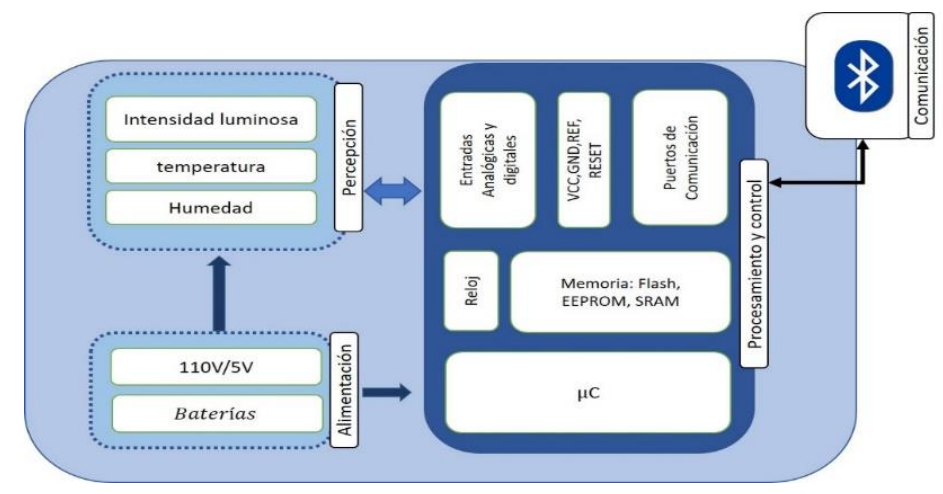

Fig. 3. Diagrama a bloques del Nodo Sensor.

\section{Nodo de Riego}

El nodo Riego (Fig. 4) obtiene información del estado de la Solución Nutritiva en el punto de concentración de esta, es decir, en el tanque de Riego en donde se realiza también el control del suministro de riego a cada módulo hidropónico a través de la activación y desactivación de las electroválvulas asignadas a cada uno.

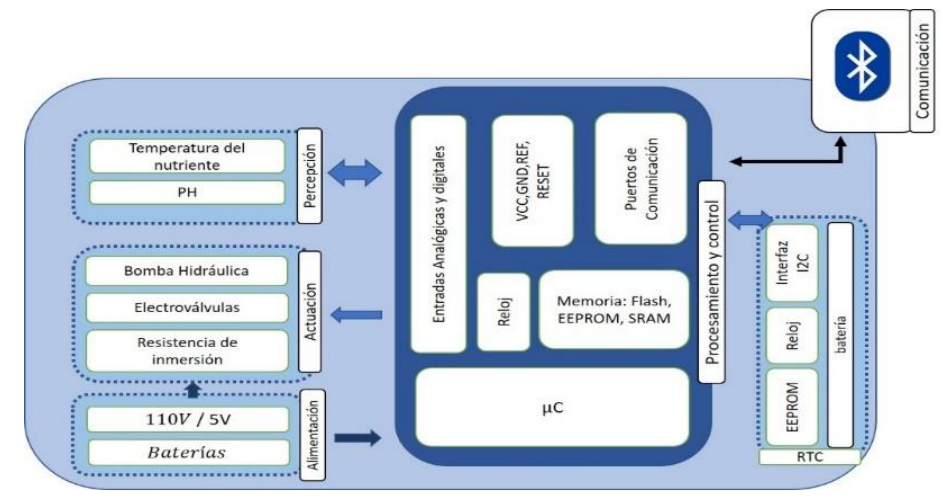

Fig. 4. Diagrama a bloques del Nodo Riego.

Dado que las condiciones de extensión dentro del invernadero pueden ser muy variadas, es decir, la cantidad de nodos dispuestos y la distancia de cada uno con respecto al servidor pueden ser diversos, una comunicación dentro del invernadero en topología 
mallada pretende subsanar dicha heterogeneidad concentrando la información en primera instancia a un nodo maestro que posteriormente reenvíe los datos al servidor.

\section{Capa de red}

En la capa de red se soporta la conectividad de los equipos, en donde son aplicados diversos protocolos y tecnologías de comunicación orientados a establecer la conexión punto a punto de los dispositivos implicados en el proyecto para realizar la transferencia de datos entre ellos. Se definió un caso mínimo de topología de red, el cual cubre las necesidades de un huerto de autoconsumo o pequeño invernadero (Fig. 5) dividiendo la solución en dos áreas de aplicación: Intra Invernadero y Extra Invernadero.

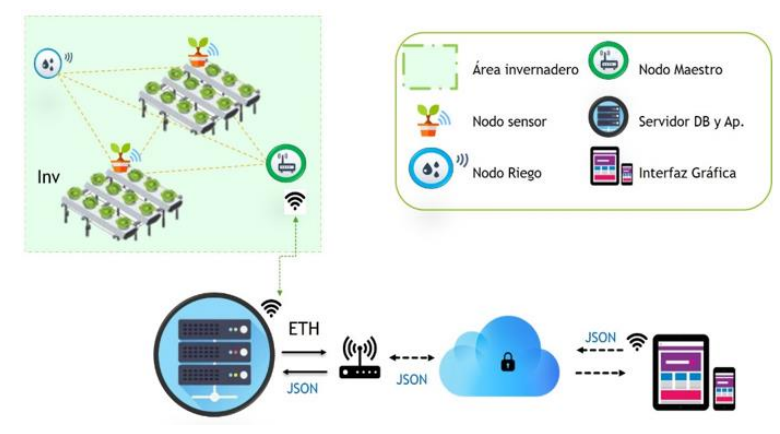

Fig. 5. Arquitectura general de red propuesta para el sistema de gestión inteligente para cultivos hidropónicos, caso mínimo.

En el área Intra-Invernadero se define la conexión entre los nodos por medio de una topología de red mallada con la finalidad de tomar ventaja de sus capacidades de área de cobertura, robustez y dinamismo. Dicha comunicación mallada es gestionada por el nodo Maestro (Figura 6), el cual asigna el identificador dinámico de los nodos, mantiene activa la red y concentra la información recaba en cada nodo.

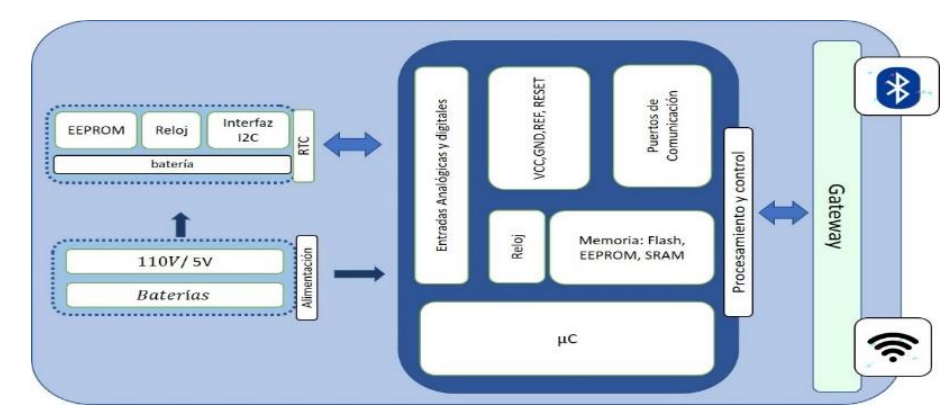

Fig. 6. Diagrama a bloques del nodo Maestro.

En el área Extra-Invernadero se considera la comunicación de la información concentrada por el nodo Maestro hacia el servidor y de la interfaz de usuario al servidor. Con la finalidad de reducir los requerimientos iniciales de instalación, el Servidor es configurado como punto de acceso inalámbrico (WAP) para proveer la comunicación 
local de los nodos maestro y de la interfaz de usuario al servidor (fig. 7), en caso de que el usuario final cuente con la infraestructura, la conexión puede ser realizada a través del protocolo Ethernet y hacer uso de sus servicios (fig. 8).

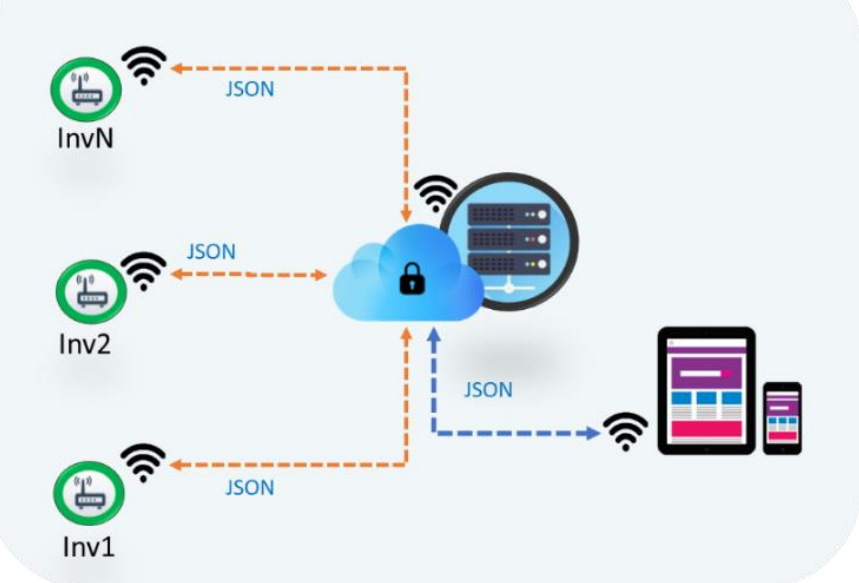

Fig. 7. Comunicación Extra Invernadero caso servidor configurado como WAP.

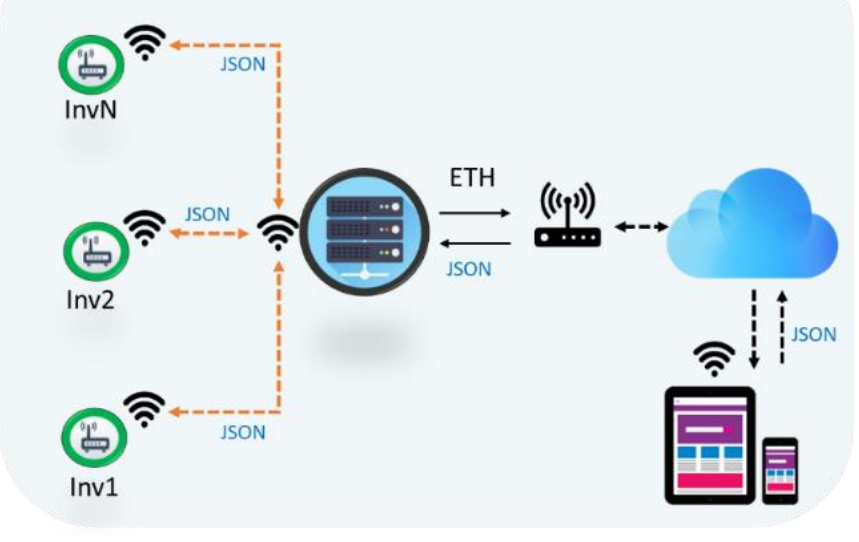

Fig. 8. Comunicación Extra Invernadero caso servidor conectado a la infraestructura del agricultor.

Dado que, el área destinada a un cultivo, el tipo de módulo hidropónico y la distribución de estos son muy diversos: entre agricultores, dentro de los mismos campos de cultivo o invernaderos, así como entre cada campaña agrícola; se definió una topología de red modular en diferentes etapas, para satisfacer las necesidades previamente mencionadas, permitiendo que el mismo modelo pueda ser implementado en diversos casos, desde huertos de autoconsumo hasta sistemas de cultivos desplegados en múltiples invernaderos. En la Fig. 9 se muestra la topología de red general de la solución. 


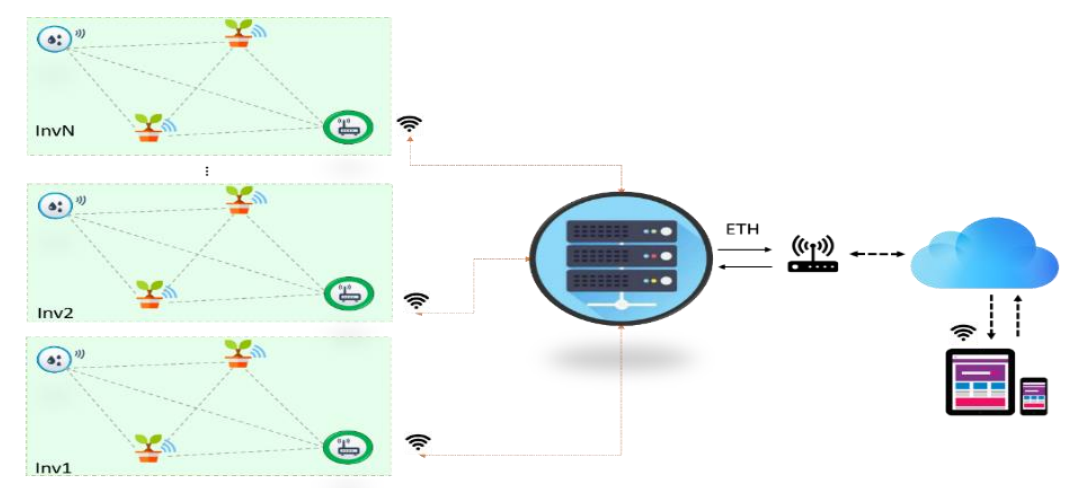

Fig. 9. Arquitectura general de red propuesta para el sistema de gestión inteligente para cultivos hidropónicos, caso múltiples invernaderos.

\subsection{Capa de procesamiento y de aplicación}

En esta capa se definieron los algoritmos de tratamiento de los datos recabados por los nodos Sensor y de Riego, mientras que en la capa de aplicación se realiza la implementación y presentación de la capa de procesamiento de datos para proveer los servicios evolucionados.

Dicho procesamiento es realizado por el Servidor (fig. 10), el cual, almacena los datos y de acuerdo con las acciones definidas, realiza la toma de decisiones de activación y desactivación de los actuadores por medio del cambio de estado del circuito de relevadores, para mantener el cultivo dentro de los valores óptimos para su desarrollo.

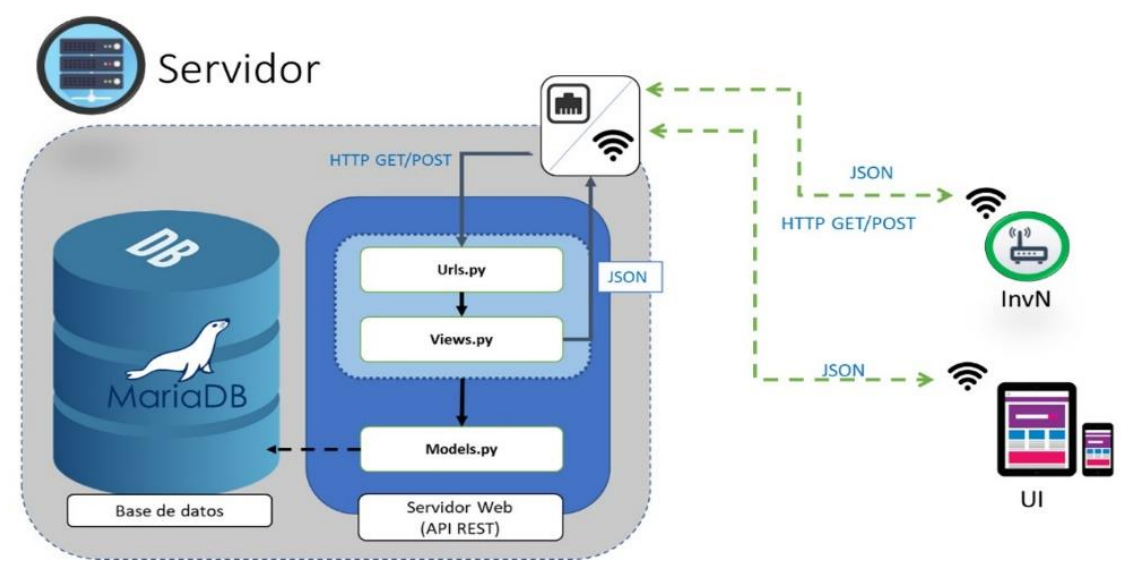

Fig. 10. Diagrama a bloques de la funcionalidad del Servidor del sistema de gestión inteligente.

\subsection{Protocolo de comunicación Pouani}

Durante el diseño del sistema de gestión basado en IoT para cultivos hidropónicos, se definió el protocolo de comunicación a nivel de aplicación "PouaniPonos", para la 
definición de la trama de comunicación de los valores obtenidos durante la fase de percepción, así como de los mensajes de configuración de los nodos. Más adelante profundizaremos en el diseño de dicho protocolo.

\section{Preprocesamiento de los datos}

Para definir la capacidad de memoria de la trama, es necesario considerar los valores mínimos y máximos que podría tener un parámetro en los diferentes casos de configuración y la precisión del dato. Por lo cual, se propuso realizar un preprocesamiento de los datos en cada nodo, con la finalidad de optimizar el espacio de memoria asignado a cada valor del parámetro obtenido en la capa de percepción.

\section{Formato de trama}

Una vez pre-procesados los valores, es necesario transmitirlos al servidor para realizar su procesamiento, almacenamiento y proporcionar los servicios. En la Fig. 11 se muestra el formato de la carga útil del nodo sensor y nodo riego en el caso de transmisión de las lecturas obtenidas de acuerdo con el formato de trama para la pila de protocolos BLE. Podemos ver que, con el diseño mínimo de la trama, se requiere 9 bytes de los 32 bytes disponibles por el transceptor nRF24L01+, por lo tanto, contamos con la capacidad de aumentar en un futuro el número de parámetros asignados a la fase de percepción dependiendo las características del proyecto.

\begin{tabular}{|c|c|c|c|c|c|c|c|c|c|}
\hline $\begin{array}{l}\text { Preámbulo } \\
1 \text { byte }\end{array}$ & \multicolumn{2}{|c|}{$\begin{array}{l}\text { Dirección } \\
3-5 \text { bytes }\end{array}$} & \multicolumn{2}{|c|}{$\begin{array}{l}\text { Campo de control de paquetes } \\
\qquad 9 \text { bits }\end{array}$} & \multicolumn{4}{|c|}{$\begin{array}{l}\text { Carga útil } \\
\text { 0-32 bytes (NRF24L01+) en BLE: 27-251 bytes }\end{array}$} & $\begin{array}{c}\text { CRC } \\
1-2 \text { bytes }\end{array}$ \\
\hline \multicolumn{2}{|c|}{ Nodo Sensor } & $\begin{array}{l}\text { Tipo } \\
1 B\end{array}$ & $\begin{array}{l}\text { No. Serie } \\
\text { 2B }\end{array}$ & $\begin{array}{l}\text { def } \\
1 \mathrm{~B}\end{array}$ & $\begin{array}{l}\text { Temp } \\
1 \mathrm{~B}\end{array}$ & $\begin{array}{l}\text { Hum } \\
1 \mathrm{~B}\end{array}$ & $\begin{array}{l}\text { Temp } \\
\text { Sol } \\
1 B\end{array}$ & $\begin{array}{l}\text { Ilum } \\
2 B\end{array}$ & $\ldots$ \\
\hline \multicolumn{2}{|c|}{ Nodo Riego } & $\begin{array}{l}\text { Tipo } \\
1 B\end{array}$ & $\begin{array}{l}\text { No. Serie } \\
2 B\end{array}$ & $\begin{array}{l}\text { def } \\
1 B\end{array}$ & $\begin{array}{l}\text { p1 } \\
1 B\end{array}$ & $\begin{array}{l}\mathrm{pH} \\
1 \mathrm{~B}\end{array}$ & $\begin{array}{l}\text { Temp } \\
\text { Sol } \\
1 B\end{array}$ & $\begin{array}{l}\text { p2 } \\
2 B\end{array}$ & ... \\
\hline
\end{tabular}

Fig. 11. Diagrama a bloques de la funcionalidad del Servidor del sistema de gestión inteligente

\section{Transmisión del paquete - Creación de la red mallada Intra-Invernadero}

Como se mencionó previamente, se establece el uso de una topología de red mallada dentro del invernadero como la adecuada para cubrir las necesidades de transmisión en dicha etapa, en específico, una red BLE mallada. Por lo cual, los nodos sensores, de riego y maestro deben ser configurados de tal forma que creen dicha red y transmitan entre sí los paquetes. El protocolo definido como "PouaniPonos" señala el procedimiento de tratamiento de la trama y establecimiento de la comunicación para el caso de sistemas de gestión agrícolas (Fig. 12).

Posteriormente se configuran en los segmentos de la memoria EEPROM los valores predeterminados de los rangos que fungen como valores extremos para el monitoreo de las variables del entorno (temperatura, humedad, iluminación, $\mathrm{pH}$, temperatura de la solución), la información de identificación del nodo (tipo de nodo y número de serie), configuración de los parámetros de control del sistema (periodos de muestreo, horarios en los que la configuración es válida y estado del nodo). 
Después, se inicia la configuración y gestión de la red mallada por el nodo maestro. El cual, asigna de forma dinámica un identificador del nodo a los nodos esclavos (nodo sensor y nodo riego), promoviendo el registro dinámico de ellos, permitiendo el ingreso y egreso de nodos a la red (movilidad de los nodos).

La conexión es actualizada periódicamente con la finalidad de mantener el enlace entre los nodos y cumplir con los siguientes pasos:

1. Establecer canales de comunicación en donde el nodo es receptor de mensajes de configuración provenientes del nodo maestro o retransmisor de mensajes de lecturas obtenidas de otros nodos sensores o nodos riego (mensajes recibidos casos $\mathrm{S}$ y $\mathrm{R}$ ).

2. Si recibe mensaje del nodo maestro (mensaje caso $\mathrm{C}$ ), los valores son almacenados en el segmento de la memoria EEPROM del microcontrolador dedicada a valor de configuración definidas por el usuario.

3. Realizar el muestreo de los valores del entorno a través de los sensores de acuerdo con el periodo de muestreo configurado.

4. Procesar los valores obtenidos de acuerdo con los rangos definidos previamente.

5. Crear la trama con la carga útil en el formato diseñado para el mensaje con destino al nodo maestro.

6. Establecer el canal de comunicación al nodo más cercano y transmitir el mensaje.

7. Regresar al paso 1.

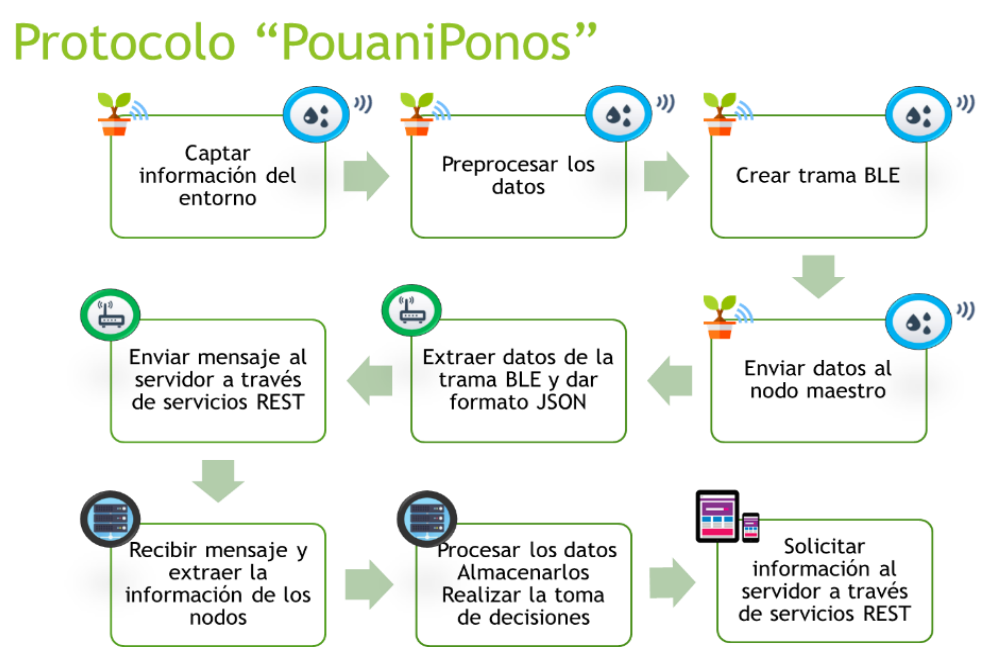

Fig. 12. Diagrama a bloques del Protocolo PouaniPonos.

\section{Extracción de datos}

Tras recibir los mensajes provenientes de los nodos sensor y de riego, el nodo maestro extrae los datos contenidos en el campo de carga útil de la trama BLE y los convierte a un objeto en formato JSON, para posteriormente transmitirlos al servidor mediante el uso de los servicios REST.

\section{Transmisión del paquete - Red de comunicación Extra-Invernadero}

Posterior a la extracción de los datos y su serialización al formato JSON, el nodo maestro requiere enviar los paquetes de mensajes al Servidor, en el cual, se establece 
una comunicación en topología estrella, la cual permite la conexión de múltiples nodos maestros al servidor. Dicha red local es proveída por el servidor, el cual es configurado como punto de acceso inalámbrico (WAP) por medio del transceptor de tecnología WiFi incorporado.

La transmisión de los paquetes se realiza a través del consumo de servicios REST con la finalidad de agregar, eliminar, consultar y configurar los valores de configuración, lecturas e información de usuario a las rutas de los recursos asignados del servidor para proveer los servicios de almacenamiento y procesamiento de los datos.

\section{Servicio de almacenamiento del servidor}

Tras recibir las peticiones de servicios REST a los URLs definidos con los datos de las lecturas proveídos por los nodos, se realiza el procesamiento de dichos valores para visualizar la lectura del nodo de acuerdo con lo sensado (es decir, previo de ser preprocesada). Posteriormente los datos son almacenados en las tablas de la base de datos del servidor de acuerdo con los modelos diseñados de la aplicación REST.

\section{Despliegue de datos en la interfaz de usuario}

Después de que el usuario realiza el inicio de sesión en la aplicación móvil, la vista inicial cumple con la función de desplegar la información de monitoreo de los cultivos, la figura 13 muestra el diagrama de vistas de la interfaz gráfica, en el cual, posterior a que una vista es llamada, se realiza el consumo de servicios REST para la consulta de los datos almacenados en la base de datos, estos son precargados y mostrados asíncronamente en la interfaz de usuario.

\section{Configuración de datos en la interfaz de usuario}

La interfaz de usuario permite peticiones de agregar, configurar y eliminar información en el servidor a través del consumo de servicios REST. Dichas peticiones se realizan a través de las URLs asignadas que a su vez dirigen a los controladores que contienen el procedimiento adecuado de tratamiento a las peticiones. Para posteriormente actualizar los datos contenidos en las tablas de la base de datos.

\section{Extracción y envío de los datos de configuración en el Servidor}

Existe un segundo tipo de mensaje que implica a los nodos dentro del invernadero, este es el mensaje de configuración asignado a través de la interfaz de usuario. Para obtenerlo, el nodo maestro realiza peticiones de consulta al servidor a través de los servicios REST, en los cuales el servidor responde con un mensaje de configuración en formato JSON, que contiene los valores de previamente preprocesados con la finalidad de reducir el tamaño de memoria asignada a cada parámetro. Extrae los datos contenidos en el mensaje en formato JSON de-serializándolo y asignando los valores a una estructura de datos con propiedades, sin métodos. 


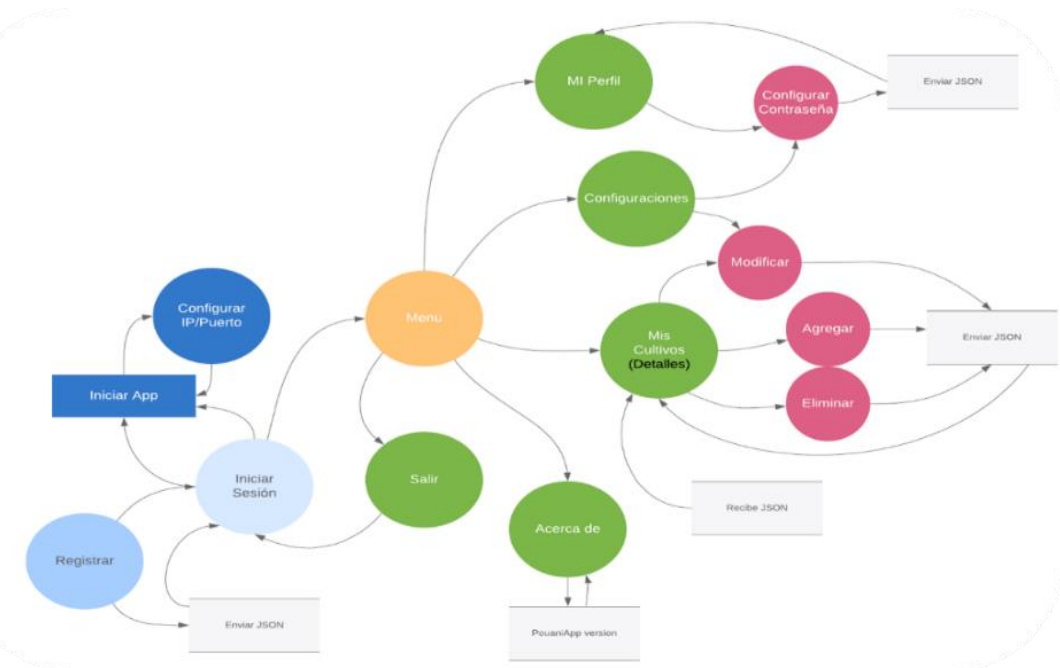

Fig. 13. Diagrama de vistas diseñadas para la interfaz gráfica de dispositivos móviles

Dichos valores se asignan a la carga útil de la trama BLE (Fig. 14) para su posterior transmisión a todos los nodos dentro del invernadero que contiene los valores máximos y mínimos de los parámetros, su estado (si están habilitados), periodo en que se tomará la muestra de sensado, hora de inicio y de fin en que la configuración es válida y en el caso del nodo riego, información referente a la actividad de riego (periodos de riego y duración), en donde se transmitirán 21 bytes de datos.

Previamente, se define en el campo de encabezado de la trama BLE el tipo de mensaje, siendo tipo "S", el mensaje de configuración para el nodo sensor y el tipo "R" el mensaje de configuración del nodo riego. Por lo que, al recibir el mensaje, los nodos leen el encabezado de la trama, específicamente el tipo de mensaje y con ello filtran el tipo de nodo destinatario de dicha configuración y después se realiza la actualización de valores de configuración en la memoria EEPROM de cada nodo.

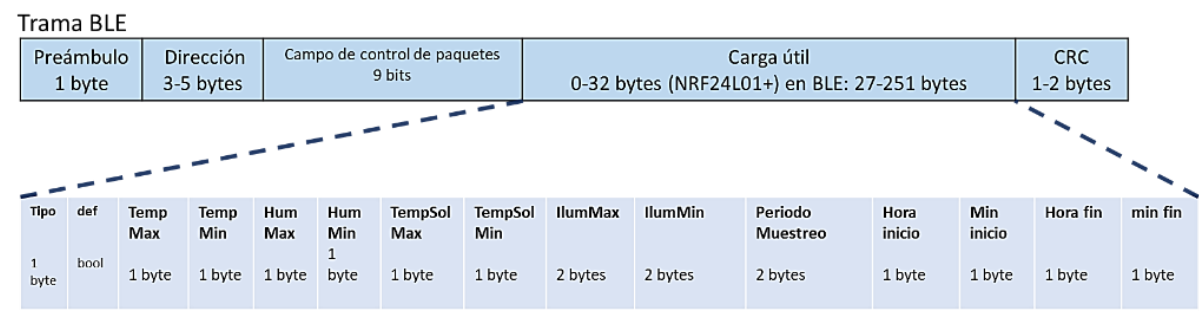

Nodo Sensor

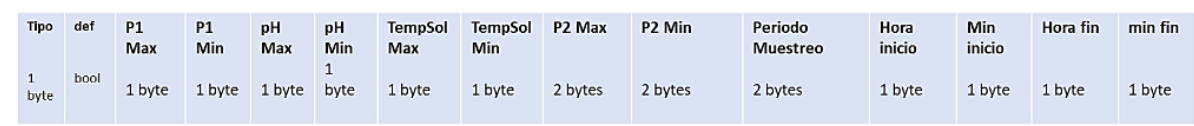

Nodo Riego

Fig. 14. Estructura de datos del mensaje de configuración de los nodos sensor y de Riego en formato BLE. 


\subsection{Configuración y comunicación con el servidor}

El servicio de base de datos para contar con un historial del estado del entorno de los cultivos (obtenidos por los nodos sensores y de riego) y el servicio web de aplicaciones REST para proveer los modelos, controladores y urls necesarios. Como se mencionó en los capítulos anteriores, la base de datos fue diseñada sobre el gestor MariaDB que permite crear modelos relacionales entre los campos definidos para la solución, mientras que los servicios REST fueron configurados sobre Django, permitiéndonos crear modelos simples y controladores para proveer las acciones diseñadas a través de diversas peticiones por medio del consumo de los servicios REST a través de una interfaz de usuario o desde los nodos maestro.

\subsection{Creación de la interfaz de usuario}

La interfaz de usuario permite la interpretación del estado del cultivo a través de una aplicación, mostrando sus variaciones a lo largo de la campaña de cultivo. El usuario puede apoyarse en ella para la toma de decisiones respecto a la activación y desactivación de los actuadores para regular dichas variables. Con ello, el riesgo de daño o abandono durante su desarrollo o periodos críticos puede disminuir y potencializa la probabilidad de obtener productos de mayor calidad y uniformidad.

Las Figs. 15 y 16 muestran las vistas de la interfaz de usuario creada para terminales móviles bajo Sistemas Operativos Android. En las cuales se cubren las necesidades de autenticación básica a través de una validación de usuario / contraseña, el despliegue de la información del estado del cultivo a través de las lecturas de los nodos y los servicios de agregar, remover, modificar la información de los cultivos y la asociación de la información de ubicación del cultivo y nodos.

De igual forma se configuraron validaciones de llenado de las formas para asegurar que el usuario llene los campos, previo a solicitar las peticiones al servidor. Así como una vista para realizar las configuraciones de la información para conectividad con el servidor de acuerdo con su dirección IP y puerto.

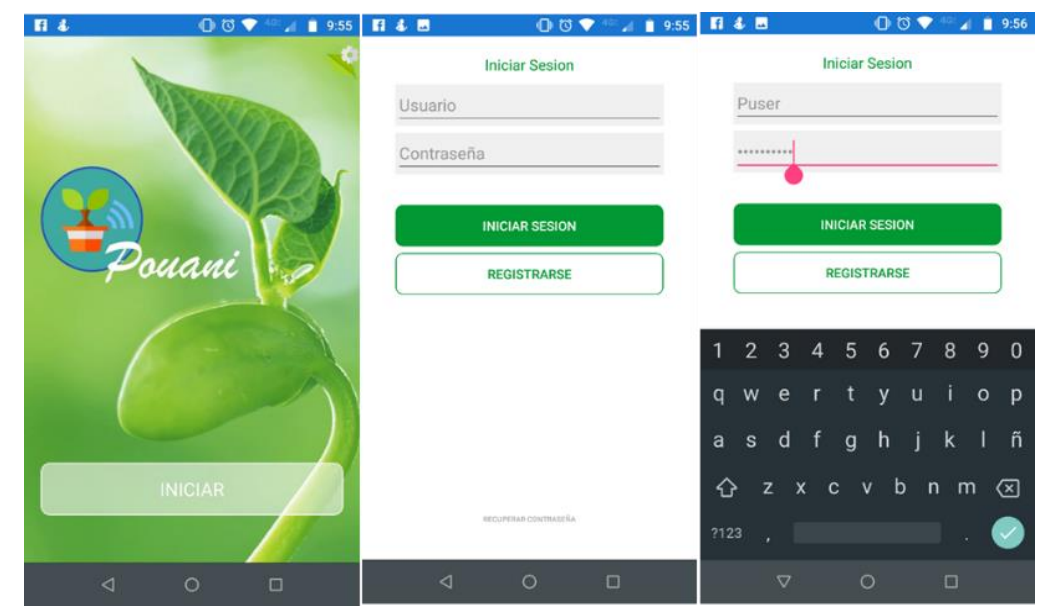

Fig. 15. Vista de Error al intentar Iniciar sesión sin usuario o contraseña (derecha) y vista del formulario de Registro de usuario 

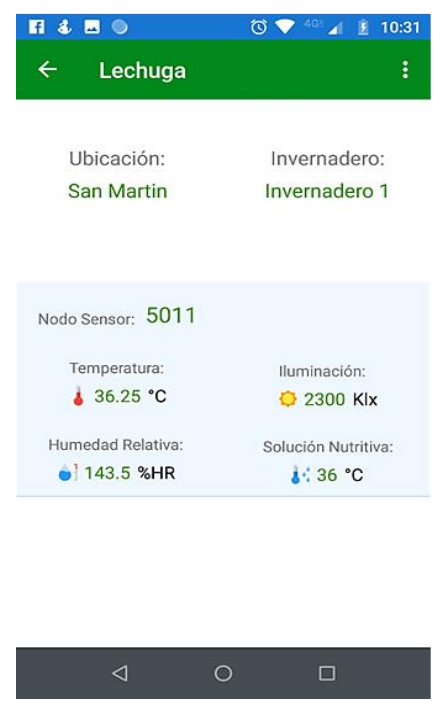

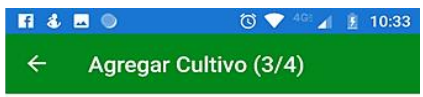

Seleccionar Invernadero Invernaderos en San Martin
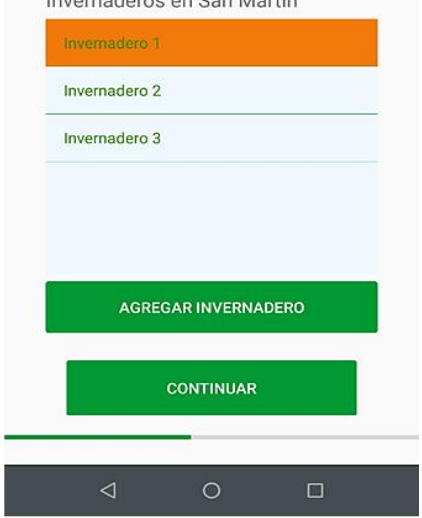

Fig. 16. Vistas de Configuración y Monitoreo.

\section{Conclusiones}

Debido al rápido crecimiento de la población y la alta demanda que esto produce, es necesario desarrollar sistemas que apoyen las necesidades básicas de los pueblos, por ello, los sistemas de agricultura inteligente han destacado ampliamente por sus aportaciones en el sector agrícola en diversos países, en donde las condiciones del entorno son poco favorables, pero el acceso a la tecnología y su implementación en los cultivos permite reducir la hostilidad y los riesgos durante el desarrollo y crecimiento de las plantas, permitiendo que los métodos agrícolas evolucionen de conocimientos empíricos a ser métodos profesionales que puedan ser medidos cualitativa y cuantitativamente.

El principal enfoque del presente proyecto es crear equipos que provean las herramientas al sector agrícola, que sean de fácil acceso y uso, con la finalidad de reducir la brecha tecnológica existente entre los productores agrícolas de los sectores mini, microempresa y de autoconsumo respecto a los productores intensivos, los cuales cuentan con una mayor capacidad adquisitiva para implementar soluciones de gestión y automatización en sus cultivos.

Con lo cual el diseño de una solución aplicable a la agricultura hidropónica requirió de múltiples esfuerzos para cubrir los principales requerimientos para dichos sistemas. El diseño modular de la solución nos permitió desarrollar equipos capaces de crecer en el sentido de la capacidad de adquisición de los datos. Esto es, a nivel de prototipo, los nodos pueden obtener la información de los parámetros clave del entorno definidos en el diseño, pero de acuerdo con las necesidades de monitoreo del agricultor, se dispone de memoria y de puertos para agregar o modificar los sensores y actuadores, permitiendo con ello crear diversos productos de monitoreo específicos a partir del diseño base de los equipos. 
La topología de red del sistema fue basada en un diseño modular y escalable, que permita el crecimiento del sistema de acuerdo con las necesidades del productor. Por lo que podemos definir el producto en un paquete de inicio que contenga los equipos mínimos para modernizar el cultivo. Este paquete inicial contiene el nodo sensor, nodo de riego, nodo maestro, servidor y aplicación móvil, los cuales proveen la gestión del cultivo para un área de cobertura de un módulo hidropónico. Posteriormente y de acuerdo con el crecimiento del sistema de cultivo o la capacidad adquisitiva del productor, éste puede obtener nodos sensores o de riego adicionales para ampliar la cobertura de monitoreo y gestión del sistema e incorporarlos sin necesidad de reconfigurar la red, siendo un proceso transparente al usuario.

Además, es claro que la tecnología avanza y con ellos los protocolos de comunicación evolucionan, por ello se diseñó un modelo flexible el cual permita, en un futuro, modificar los protocolos de comunicación a tecnologías más recientes con afectaciones mínimas para el usuario.

La definición del protocolo de formato y tratamiento de la trama a nivel aplicación "PouaniPonos" pretende normalizar los métodos de comunicación y tratamiento de los datos para sistemas enfocados a la agricultura inteligente. Las ventajas del protocolo de trama "PouaniPonos" permite la optimización del espacio de memoria asignado a la carga útil de la trama a través del preprocesamiento de los datos recabados y con ello aprovechar dicho espacio de memoria para la transmisión de múltiples parámetros por nodo. Así mismo, el diseño de la topología de red híbrida que aprovecha las fortalezas de las tecnologías de comunicación WiFi y Bluetooth para entornos agrícolas, demostró ser viable en condiciones de laboratorio. De acuerdo con la tendencia de comportamiento y respuesta en términos de funcionalidad, el siguiente paso en el desarrollo del sistema, es implementar la solución y pruebas en campo, con la finalidad de complementar el análisis y resultado del comportamiento de los equipos en condiciones reales.

Finalmente, se plantea un modelo general de cultivos hidropónicos. El cual, se espera presente una base para futuros sistemas para agricultura inteligente, basados en tecnologías de nueva generación, los cuales eventualmente permitan a la mayoría de la población, contar con una fuente de alimento sustentable, eficiente y de mejor calidad mediante el uso de sistemas de cultivos hidropónicos personales.

\section{Referencias}

1. Servicio de Información Agroalimentaria y Pesquera (SIAP): Uso de tecnología y servicios en el campo Cuadros tabulares (2016)

2. World Bank, CIAT, CATIE: Climate-smart agriculture in Mexico. CSA country profiles for Latin America Series. World Bank Gr (2014)

3. Oasis, S.: Manual de hidroponia. 32 (2002)

4. Patokar, A.M., Gohokar, V.V.: Precision Agriculture System Design Using Wireless Sensor Network. pp. 169-177, doi: 10.1007/978-981-10-5508-9_16 (2017)

5. Verdouw, C.: Internet of Things in agriculture. CAB Rev Perspect Agric Vet Sci Nutr Nat Resour 11: doi: 10.1079/PAVSNNR201611035(2016)

6. Kiani, F., Seyyedabbasi, A. Wireless Sensor Network and Internet of Things in Precision Agriculture. Int J Adv Comput Sci Appl 9, 99-103 doi: 10.14569/ijacsa.2018.090614 (2018)

7. Azcón-Bieto, J., Talón, M.: Fundamentos de fisiología vegetal (2003)

8. Araceli, M.: Manual de hidroponia, 1a Ed. Instituto de Biología, Distrito Federal (2014) 
Propuesta de sistema de gestión inteligente basado en IoT para hidroponia

9. Minerva, R., Biru, A., Rotondi, D. Towards a definition of the Internet of Things (IoT). IEEE Internet Things 86. doi: 10.5120/19787-1571 (2015) 